Yau, D. M., Yokoyama, N., Goshima, Y., Siddiqui, Z. K., Siddiqui, S. S., and Kozasa, T. (2003). Identification and molecular characterization of the $G$ alpha12-Rho guanine nucleotide exchange factor pathway in Caenorhabditis elegans. Proc. Natl. Acad. Sci. USA 100, 14748-14753.

Zipperlen, P., Fraser, A. G., Kamath, R. S., Martinez-Campos, M., and Ahringer, J. (2001). Roles for 147 embryonic lethal genes on C. elegans chromosome I identified by RNA interference and video microscopy. EMBO J. 20, 3984-3992.

\title{
[4] High-Throughput RNA Interference Screens in Drosophila Tissue Culture Cells*
}

\author{
By Susan Armknecht, Michael Boutros, Amy Kiger, \\ Kent Nybakken, Bernard Mathey-Prevot, and \\ NORBERT PERrimon
}

\begin{abstract}
This chapter describes the method used to conduct high-throughput screening (HTs) by RNA interference in Drosophila tissue culture cells. It covers four main topics: (1) a brief description of the existing platforms to conduct RNAi-screens in cell-based assays; (2) a table of the Drosophila cell lines available for these screens and a brief mention of the need to establish other cell lines as well as cultures of primary cells; (3) a discussion of the considerations and protocols involved in establishing assays suitable for HTS in a 384-well format; and (A) a summary of the various ways of handling raw data from an ongoing screen, with special emphasis on how to apply normalization for experimental variation and statistical filters to sort out noise from signals.
\end{abstract}

\section{Introduction}

A full grasp of the biological underpinnings of an organism such as Drosophila requires not only knowledge of the inherent function, expression profile, and subcellular localization of every gene product in the organism but also an awareness of the dynamic range of each gene product's interactions with other gene products over time and development. Although much information has accrued from the application of classical genetics, biochemistry, and developmental biology, the time and labor involved in these approaches makes their use in whole-genome assays a distant possibility. The completion of the Drosophila genome sequence in

*S. A., M. B., and A. K. contributed equally to this work. 
2000 (Adams et al., 2000) ushered the possibility of developing genomewide approaches to systematically explore gene function in this organism. Newly emerged technologies were quickly exploited to extract maximal information encrypted in the raw sequence of the Drosophila genome. Among them, RNA interference (RNAi) is proving to be immensely valuable. RNAi refers to the ability of double-stranded RNA (dsRNA) or short-interfering RNA (siRNA) to silence a target gene through the specific destruction of the gene's mRNA. Unlike other genomic-based approaches such as microarrays and proteomics, RNAi provides a direct link from gene to function and, to date, offers the best tool for realizing the full potential of the genome project. The mechanisms by which RNAi silences gene expression are not discussed here as they can be found in a series of excellent reviews (Dykxhoorn et al., 2003; Fire, 1999; Hannon, 2002; McManus and Sharp, 2002; Paddison and Hannon, 2003). Here we describe the method used to conduct high-throughput screening (HTS) RNAi in Drosophila tissue culture cells. The concept is based on the seminal observation by Clemens et al. (2000) that the single addition of long dsRNAs to Drosophila cells leads to an efficient RNAi effect.

\section{Platforms Used for High-Throughout Screening (HTS) RNA Interference (RNAi) Screens}

We have established a facility to support the investigation of a widerange of questions in cell biology through large-scale RNAi screens in Drosophila cells [Drosophila RNAi Screening Center (DRSC); http:// flyrnai.org]. Our long-term goal is to build a comprehensive database of genetic network interactions, protein-protein interactions, cellular phenotypes, and a catalog of gross cellular morphologies that can be linked to particular genes or pathways. In collaboration with the laboratory of Renato Paro and his colleagues (Hild et al., 2003), we have generated a genomewide library of $>21,000$ dsRNAs that target nearly all $(>91 \%)$ of the Drosophila predicted open reading frames for use in HTS (Boutros et al., 2004). To take advantage of this library, we developed the methodology described in Fig. 1 to rapidly identify all the genes that affect a biological outcome as measured in a specific assay.

1. First, gene-specific dsRNAs are aliquoted into unique wells of multiple 384-well assay plates by using robotics. The concentration of dsRNA in the wells ranges from 25 to $75 \mathrm{n} M(\sim 0.2 \mu \mathrm{g}$ of 500-bp length), a level sufficient with the RNAi bathing method to diminish or deplete endogenous mRNA levels (lesser dsRNA needed in the RNAi transfection method). 
2. Cells are uniformly dispensed into the dsRNA-containing 384-well assay plates, using a Multidrop ${ }^{\circledR} 384$ (Thermo Electron Corporation, Ontario, Canada) liquid dispenser at $1 \times 10^{4}$ to $3 \times 10^{4}$ cells per well, depending on the cell line and assay time course. For RNAi treatment by the bathing method, cells are first exposed to the dsRNAs in serum-free medium. After $\geq 30$ min in serum-free medium, the Multidrop 384 is used again to add additional culture medium containing serum.

3. After the appropriate incubation time, cells can be either exposed first to a specific treatment or induction or directly processed for the assay readout.

The plate reader and automated microscopy are two common detection methods for data acquisition from the 384-well plates. The plate reader quantifies relative luminescence or fluorescence levels to a single numerical readout. The measurement represents the sum signal of all cells per well and is often determined in a biochemical assay performed on lysed cells. Plate reader screens provide a quantitative set of data amenable to normalization and various statistical analyses. At the DRSC, we use the

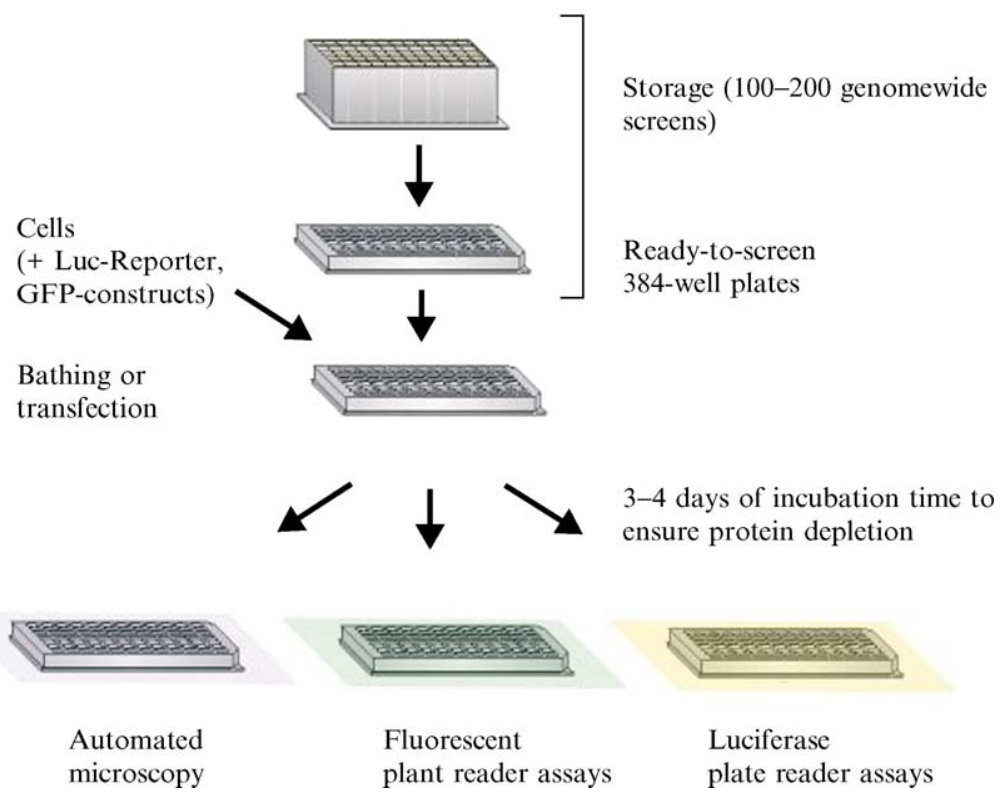

FIG. 1. RNAi screens in cell-based assays. There are three major steps: (1) array gene-specific dsRNAs into 384-well assay plates, (2) add cells to assay plates and incubate, and (3) perform assay and measure readout with plate reader or monitor by microscopy. 
Analyst $^{\text {TM }}$ GT (Molecular Devices, Sunnyvale, CA) plate reader. It has luminescence, fluorescence intensity, fluorescence polarization, time-resolved fluorescence, and absorbance detection modes in 96-, 384-, and 1536-well formats. A 384-well plate can be read in $1 \mathrm{~min}$, providing rapid screening. It is also equipped with a bidirectional stacker for unattended operation. An application of the plate reader to quantify signals can be found in the assay conducted by Boutros et al. (2004), in which levels of luciferase activity were measured in each well and used as a linear measure of total cell number per well.

The inverted fluorescent microscope with automated acquisition software (MetaMorph ${ }^{\circledR}$, Universal Imaging, Downingtown, PA), is used to track, focus, and capture fluorescent images of the cells within each well across an entire plate, providing cell-by-cell data in image fields of either live or fixed cells. The microscopy-based screens are more qualitative at present and are valuable for detecting inherent spatial information relevant to particular cell biological processes (i.e., protein localization, vesicular trafficking, axonal outgrowth, or host-pathogen interactions). Based on this approach, Kiger et al. (2003) designed an assay that followed fluorescently labeled actin filaments and microtubules to monitor cytoskeletal organization, cell attachment, cell spreading, and cell shape. In our screens, we have used the Discovery-1 ${ }^{\mathrm{TM}}$ (Molecular Devices) high-content screening system. It has automated filter and dichroic wheels and a six-objective turret with high-speed laser auto-focus; it can measure up to eight fluorophores per assay in multiwell plates. In addition, the Discovery-1 is equipped with CataLyst Express ${ }^{\mathrm{TM}}$ (Thermo Electron Corporation) robotic arm for continuous, unattended operation. Depending on the assay, commercially available software can extract quantitative information on fluorescence intensity and distribution on a cell-by-cell basis or of the entire field. Automated image analysis is not yet generally applicable to all screens, and this is clearly an area that needs further development.

With both the plate reader- and microscopy-based platforms, there are two current methods for RNAi that involve either bathing or transfecting cells with dsRNAs. In the first method, cells are directly dispensed into 384-well plates containing dsRNA, which is then taken up by the cells (presumably by macropinocytosis), and incubated together for 3-5 days before the assay is read (bathing condition). The second method (transfection condition) is used when the design of the assay dictates that cells be transfected with a reporter gene and normalization constructs, and/or with expression vectors for a particular component of a signaling pathway or cellular process. Detailed protocols for both conditions are found in the section "Designing RNAi screens in HTS Format." 


\section{Drosophila Cells Available for RNAi Screens}

We have collected a number of Drosophila cell lines, but have worked mostly with S2, S2R+, Kc, and clone 8 (cl8) cells in our screens. Table I lists the cell lines available in our laboratory and summarizes their salient characteristics. A number of additional cell lines are also available from DGRC (http://dgrc.cgb.indiana.edu).

Although the number of Drosophila cell lines currently available is relatively small, we expect that the next few years will witness the development of additional cell lines. In this regard, there are ongoing efforts to establish cultures of primary myoblastic, neuronal, and glial cells as well as hemocytes (insect hematopoietic cells) to be used in RNAi high-throughput screens. These efforts are not detailed in this chapter, but we mention two future applications that stress their importance and highlight the unique advantage of using Drosophila as an organism. The vast collection of mutant embryos that exist in Drosophila can serve as a valuable resource for establishing cell lines with sensitized background with which to perform HTS RNAi enhancer or suppressor screens. In addition, the availability of an extensive collection of embryos in which endogenous proteins have been tagged with GFP after exon trapping (Kelso et al., 2004) can be exploited to establish cell lines to conduct visual screens.

\section{Designing RNAi screens in HTS Format}

\section{Considerations}

To develop an HTS, a cell-based assay must be adapted to high-density cell culture plate formats. (We routinely use 384-well plates rather than 96-well plates as they require a lesser amount of dsRNA.) Different types of plates are available, depending on the assay detection method. For example, white, solid-bottom plates are used for luminescence assays and black, clear-bottom plates for fluorescence assays. Other formats are possible in future screens once the technology is sufficiently advanced. For example, the use of high-density spotted arrays of dsRNAs on glass slides would substantially reduce materials necessary for genomewide screens (Carpenter and Sabatini, 2004). The following is a checklist of important considerations to weigh before embarking on a high-throughput RNAi screen:

\section{Choices for Cell Lines}

1. Will existing cell lines or primary cells be used?

2. Does RNAi by bathing or transfection work in the specific cells?

3. Do cells express the target and how refractory is it to RNAi? 
TABLE I

List of Drosophila Cell Lines Available for High-Throughput Screening (HTS) RNA Interference (RNAi)

\begin{tabular}{|c|c|c|c|}
\hline Line & Origin & Characterisitics & Reference \\
\hline Schneider's Line S2 (S2) & $\begin{array}{l}\text { Dissociated embryos, } \\
\quad \text { near hatching (Oregon } R)\end{array}$ & $\begin{array}{l}\text { Hemocyte-like gene expression, } \\
\text { phagocytic, semiadherent in } \\
\text { colonies, round, granular cytoplasm }\end{array}$ & Schneider, 1972 \\
\hline Schneider's S2 (S2*) & $\begin{array}{l}\text { Dissociated embryos, } \\
\quad \text { near hatching (Oregon } R)\end{array}$ & $\begin{array}{l}\text { Subclone of S2 cells that is more } \\
\text { responsive to LPS, particularly } \\
\text { after priming with } 1 \mu \mathrm{M} \\
\text { 20-hydroxy ecdysone }\end{array}$ & Schneider, 1972 \\
\hline Schneider's S2 (S2C) & $\begin{array}{l}\text { Dissociated embryos, } \\
\quad \text { near hatching (Oregon R) }\end{array}$ & $\begin{array}{l}\text { Hemocyte-like gene expression, } \\
\text { semiadherent in colonies, round, } \\
\text { granular cytoplasm }\end{array}$ & Schneider, 1972 \\
\hline Schneider's S2 (S2-R+) & $\begin{array}{l}\text { Dissociated embryos, } \\
\quad \text { near hatching (Oregon } R \text { ) }\end{array}$ & $\begin{array}{l}\text { Hemocyte-like gene expression, } \\
\text { phagocytic, adherent, flat cells, } \\
\text { Fz+ and Wg-responsive }\end{array}$ & $\begin{array}{l}\text { Schneider 1972; } \\
\text { Yanagawa, } \\
\text { et al., } 1998\end{array}$ \\
\hline Schneider's S2 (DL2) & $\begin{array}{l}\text { Dissociated embryos, } \\
\quad \text { near hatching (Oregon } R \text { ) }\end{array}$ & $\begin{array}{l}\text { Hemocyte-like gene expression, } \\
\text { phagocytic, adherent monolayer } \\
\text { of uniformly round, smooth cells }\end{array}$ & Schneider, 1972 \\
\hline Schneider's Line S3 & $\begin{array}{l}\text { Dissociated embryos, } \\
\quad \text { near hatching (Oregon } R \text { ) }\end{array}$ & $\begin{array}{l}\text { Adherent, spindle-shaped cells, } \\
\text { ecdysone responsive, grow } \\
\text { in clumps }\end{array}$ & Schneider, 1972 \\
\hline Kc (Kc 167) & $\begin{array}{l}\text { Dissociated embryos, } 8-12 \mathrm{~h} \\
\quad(\mathrm{~F} 2 \text { ebony } \times \text { sepia })\end{array}$ & $\begin{array}{l}\text { Hemocyte-like gene expression, } \\
\text { phagocytic, uniformly round, } \\
\text { clump in sheets, ecdysone } \\
\text { responsive into adherent, } \\
\text { bipolar spindle-shaped cells }\end{array}$ & $\begin{array}{l}\text { Echalier and } \\
\quad \text { Ohanessian, } 1969\end{array}$ \\
\hline
\end{tabular}


1(2)mbn

ML-DmBG2

ML-DmBG6

Clone 8
Third-instar larvae tumorous hemocytes, [l(2)malignant blood neoplasm]

Dissociated third-instar larvae brain and ventral ganglia ( $y \vee f$ mal $)$

Dissociated third-instar larvae brain and ventral ganglia $(y \vee f m a l)$

Third-instar larvae wing imaginal discs
Larger cells, larger granular, complex cytoplasm, phagocytic, aneuploid, heterogenous size and shape

Acetylcholine, HRP expression, neuronal-like processes

Acetylcholine, HRP expression, neuronal-like processes

Derived from wing columnar epithelial cells, adherent, will form multiple layers, conserved signaling pathways
Haars et al., 1980

Ui et al., 1994

Ui et al., 1994

Peel et al., 1990 
4. Do cells need to be transfected to express a specific gene product that can serve as a target, modifier, or readout of the pathway or process of interest?

5. Is the phenotype or outcome easily detected in the chosen cell line?

6. Is this phenotype or outcome uniform across the cell population?

Assay Optimization with Genes Known to Belong to a Pathway or a Cellular Process

1. Select a few positive and negative control dsRNAs and confirm the desired phenotype or appropriate biological readout in response to RNAi.

2. When possible, confirm depletion of the gene product after RNAi of the positive and negative controls by immunoblotting or immunofluorescence.

3. Estimate the robustness of the assay in identifying the desired phenotype or outcome.

4. Familiarize yourself with the range of experimental variability in the assay.

5. Determine the optimal range of dsRNA concentration resulting in most penetrant and uniform phenotypes.

6. Determine optimal RNAi incubation time and plating density.

\section{Configure the Assay to the 384-Well Plate Format}

Plates. Appropriate plates depend on the type of platform used to read the assay. Confirm that the assay is compatible with these plates. Test and optimize plating, aging, staining, and detection in the 384-well plates to be used in the assay.

- Black, optically clear bottom plates (VWR No. 29444-078, Costar) are used for autoscope- and fluorescence plate reader-based assays.

- White, solid-bottom plates (VWR No. 29444-090, Costar, VWR No. 62409-072, Nunc) are used for luciferase plate reader-based assays.

- Solid black plates are used for fluorescence plate reader screens.

Imaging. Cells are screened with a $20 \times$ objective. In general, this resolution offers the best compromise: it facilitates autofocusing and yields a sufficient number of cells per field of study while being high enough to capture meaningful details in cell images. At this resolution, up to 16 sites within each well (covering the entire well surface) can be imaged; typically, images from two sites are collected. Drosophila cells are relatively small $(10-50 \mu \mathrm{m})$, and subtle differences may not be easily resolved with a $20 \times$ objective. Depending on the phenotype scored, different resolutions may need to be optimized. When using an automated 
imaging system, check whether there is a reasonable field of cells within the region imaged with a $20 \times$ objective. Cell density also impacts the autofocusing process, which can in turn interfere with proper detection of cellular phenotypes. In addition, cell density can alter cellular phenotypes. A crisp, planar, bright marker is required for fast and optimal autoscope focusing. Multiple channels can be sequentially imaged (most often a maximum of three). Test positive control dsRNA in 384-well plates to ensure that the resulting RNAi phenotype can be blindly identified among negative controls.

Check Signal/Noise and Well-to-Well Variability. Two important issues to consider during assay development are the signal/background ratio (S/B), and the well-to-well or experimental variability (EV). As assay variability increases, the $\mathrm{S} / \mathrm{B}$ ratio must increase for the screen to be successful. We recommend preparing a positive control to determine the $\mathrm{S} / \mathrm{B}$ ratio. To determine EV, add reagents to a plate with the same equipment used in the screen. Spike several wells with a positive control, and determine whether it can be reproducibly detected above the well-to-well variation. This information will give an indication of the false-positive and false-negative rate of the specific assay.

\section{Protocols for Bathing and Transfection Conditions}

The protocols have been established for cells dispensed in 384-well plates (used in the HTS RNAi screen). RNAi experiments may be done in plates of a different size, the only difference being that the amounts of reagents listed must be scaled up or down as required.

\section{Bathing Conditions for 384-Well Plates}

1. Remove 384-well plates prealiquoted with dsRNA from freezer to thaw. The 384-well plates contain $5 \mu \mathrm{l}$ of $\sim 0.05 \mu \mathrm{g} / \mu \mathrm{l}$ dsRNA in water for $\sim 0.25 \mu \mathrm{g}$ dsRNA/well.

2. Spin plates at $\sim 1200 \mathrm{rpm}$ for $1 \mathrm{~min}$ before removing seals.

3 . Count cells and then spin to pellet ( 1200 rpm, $5 \mathrm{~min})$.

4. Resuspend cells at $5 \times 10^{6}$ cells $/ \mathrm{ml}$ in serum-free medium.

5. Plate $\sim 10^{4}$ cells $10 \mu \mathrm{l} /$ well in 384 -well plates.

6. Incubate dsRNA with cells at RT for $30 \mathrm{~min}$ or more.

7. Add $30 \mu \mathrm{l}$ of complete medium to each well.

8. Seal or incubate plates in a humid chamber to prevent evaporation and minimize edge effects.

9. Incubate for 3-5 days and analyze. Length of incubation may vary depending on assay. 
TABLE II

Transfection Set-up for 384- and 96-Well Plates

\begin{tabular}{|c|c|c|c|c|c|c|}
\hline Plate & $\begin{array}{l}\text { Diameter } \\
(\mathrm{mm})\end{array}$ & $\begin{array}{l}\text { Area } \\
\left(\mathrm{mm}^{2}\right)\end{array}$ & $\begin{array}{l}\text { Fold diff. in } \\
\text { area from } \\
96 \text { well }\end{array}$ & $\begin{array}{l}\text { Fold diff. } \\
\text { in area } \\
\text { from } 6 \text { well }\end{array}$ & $\begin{array}{c}\text { DNA } \\
\text { per well } \\
(\mu \mathrm{g})\end{array}$ & $\begin{array}{c}\text { Cells per } \\
\text { well }\left(\times 10^{4}\right)\end{array}$ \\
\hline 384 & 3.5 square & 12.25 & $1 / 2.6$ & $1 / 80$ & $0.050-0.175$ & $1-3 \times 10^{4}$ \\
\hline 96 & 6.4 & 32 & 1 & $1 / 30$ & $0.20-0.30$ & $3-8 \times 10^{4}$ \\
\hline Plate & $\begin{array}{l}\text { Diameter } \\
\quad(\mathrm{mm})\end{array}$ & $\begin{array}{c}\text { Area } \\
\left(\mathrm{mm}^{2}\right)\end{array}$ & $\begin{array}{c}\text { EC buffer } \\
(\mu \mathrm{l})\end{array}$ & $\begin{array}{c}\text { Amount } \\
\text { enhancer } \\
(\mu \mathrm{l})\end{array}$ & $\begin{array}{c}\text { Amount } \\
\text { effectene } \\
(\mu \mathrm{l})\end{array}$ & $\begin{array}{c}\text { Medium } \\
\text { (with cells) } \\
\text { added to } \\
\text { well }(\mu \mathrm{l})\end{array}$ \\
\hline 384 & 3.5 & 12.25 & 8.75 & 1.0 & 0.30 & $30-50$ \\
\hline 96 & 6.4 & 32 & 21.5 & 2.5 & 0.75 & $100-150$ \\
\hline
\end{tabular}

Note: Transfection conditions in 96- and 384-well plates for HTS RNAi screening. Differences in DNA quantity, cell number, and transfection reagent amounts are scaled to well area. See text for details.

Transfection Conditions for 384-Well Plates. This protocol describes the method we have developed to screen in cell lines that require transfection of the dsRNA or for screens involving transient transfection of a transcriptional reporter (or any another construct). This approach was designed to screen for new components of signaling pathways (based on the activation of a luciferase transcriptional reporter), but it can be adapted to survey other phenomena, such as protein-protein interactions, using green fluorescent protein (GFP) fluorescence resonance energy transfer (FRET) constructs. For screening 384- or 96-well plates, it is best to automate as many steps as possible. Good dispensing machines are critical to a smooth running screen, and different dispensing machines are used for different purposes. We use the MultiDrop to aliquot transfection mixes and cells to wells. The MultiDrop relies on a peristaltic pump system and interchangeable heads to dispense fluids, which we find to be easier to keep sterile than the piston pump systems. We use the $\mu$ Fill ${ }^{\mathrm{TM}}$ (Bio-Tek, WinoOSRI, VT), piston pump machine for the luciferase assays. It is more precise for assays that are highly sensitive to slight variations in volumes across wells. (Although this is important for luciferase assays, immunostaining procedures are much less sensitive to small volume variations.) When using these machines in a highly automated procedure, it is helpful 
to familiarize oneself with their use before setting up assay plates. When using 384-well plates, we recommend testing the MultiDrop by aliquoting some of the wash solution into a blank plate as the MultiDrop can occasionally have problems when shifting from the even to the odd rows. Finally, we use Effectene ${ }^{\mathrm{TM}}$ (Qiagen, Valencia, CA) as the transfection reagent because it is a good compromise between high efficiency of transfection and relative ease of use (Table II).

1. Count cells and dilute them in fresh medium so that the desired number of cells is in 30-40 $\mu \mathrm{l}$ of medium, which can be dispensed in each well. Fill a sterile container with all the cells/medium needed plus $10 \mathrm{ml}$ to account for priming of the dispensing head. Mix well before dispensing.

2. Centrifuge the plates at $1200 \mathrm{rpm}$. Remove the sealing membranes and then add $75 \mathrm{ng}$ of control dsRNA in $5 \mu \mathrm{l}$ to the dedicated empty well in the 384-well plate.

3. Prepare EC + DNA master mix. The master mix for a 384-well plate consists of all DNA constructs that need to be introduced into cells (which may include the experimental reporter, a ligand expression vector, and the control reporter) and EC buffer, a component of the Effectene kit. Dilute the DNA in $8.75 \mu \mathrm{l} \mathrm{EC} \mathrm{buffer.} \mathrm{It} \mathrm{is} \mathrm{best} \mathrm{if} \mathrm{the} \mathrm{volumes} \mathrm{of} \mathrm{all} \mathrm{DNA}$ solutions represent less than $3 \%$ of the final volume of the master mix. If this is not possible, we recommend precipitating the DNA material and resuspending it directly in EC buffer. It is important to keep the nucleic concentration equal in all wells and use no more than $175 \mathrm{ng}$ of total nucleic acid (DNA + dsRNA) per 384-well plate. Mix EC + DNA well by inversion or pipetting.

4. Prepare the MultiDrop to dispense both transfection solution and cells. This will require two different dispensing cassettes for the MultiDrop: one exclusively used for cells and one for reagents. For the cell cassette only, wash with $70 \%$ ethanol to sterilize. Then wash both cassettes thoroughly by running sterile water and PBS through them. Always wash the cassettes with sterile water before aliquoting reagents or cells. Put the reagent cassette in the machine and set the cell cassette aside in the hood until needed. Check that the number of columns to be aliquoted is correct and then set the volume of media to be dispensed per well. This protocol uses $10 \mu \mathrm{l}$ of transfection solution per well.

5. Add enough enhancer to the EC + DNA master mix such that each well receives the equivalent of $1.0 \mu \mathrm{l}$ of enhancer from the transfection solution. Mix well by inversion. Incubate at RT for 2-3 min.

6. Complete the transfection solution by adding enough Effectene to the enhancer + EC + DNA master mix such that finally $0.3 \mu \mathrm{l}$ of Effectene is present in each well. Mix by pipetting up and down 5-10 
times or vortexing on high for $10 \mathrm{sec}$. Immediately begin dispensing the transfection solution into the assay plates, using as little of the transfection solution as possible to prime the dispensing cassette. Vortex the plate on a low setting for 5-10 sec. using a plate adapter to mix the dsRNA and the transfection solution.

7. Let the dsRNA and transfection solution incubate in the well for 4-8 min. Meanwhile, substitute the cell cassette for the reagent cassette and briefly wash the cell cassette with $10-20 \mathrm{ml}$ cell culture medium and empty the cassette after the wash. After 4-8 min, dispense the desired number of cells in the predetermined volume of medium into the 384-well plate. It is critical to get the transfection solution and the cells into the wells within $20 \mathrm{~min}$ of making the master transfection solution or the transfection efficiency well begin to fall rapidly.

8. Incubate the plates in a humidified chamber in a TC incubator for $3-5$ days, and then conduct assay.

\section{Standardization of Reporter Gene Assays}

Normalization is the process by which raw data returned from an assay is standardized relative to an internal control. This ensures that the quantitative readout is not influenced by well-to-well variability. In this section, we discuss the need for normalization in HTS RNAi transfection screens and describe some of the control luciferase reporter genes that we have developed to carry out normalization for transfection assays in a 384-well plate format.

Transfection assays for HTS RNAi screens require more careful design than for bathing assays because of the higher number of variables involved. Although it is possible to transfect materials other than nucleic acids into cells, the principal application of transfection in HTS screens is to introduce reporter genes designed to reflect the amplitude of a response to a stimulus or a cellular perturbation. Reporter constructs generally consist of an expression construct in which an indicator gene (encoding, for instance, luciferase, GFP, or CAT) is placed under the transcriptional control of promoter and enhancer elements that modulate the indicator gene expression in response to the stimulus or perturbation under study. The strength of the signal measured in such an assay will reflect the biological outcome (i.e., the response to a stimulus), but will also depend on the number of cells successfully transfected with the reporter gene and the strength of expression of the transfected indicator gene. As transfection of cell populations is neither uniform in terms of the percent of cells transfected nor in terms of the amount of material that is introduced 
into each cell, some method of standardization is required to control for these variables.

Ideally, a standardization control will provide information about both the number of cells that have taken up the transfected material as well as the particular cell type's ability to express the transfected gene. In practice, however, it is difficult to separate these two variables and they are treated as one variable, often referred to as the transfection efficiency. If the variation in transfection efficiency is sufficiently low, a standardization control can also provide information on the number of transfected cells present in a well. Just as it is important in HTS RNAi screens to design an experimental reporter that leads to a high signal-tonoise ratio in response to a stimulus, it is equally critical to have a robust control reporter that accurately reflects the transfection efficiency within each well. We have found that cotransfection of both experimental and control reporters, followed by sequential assay of the two reporters, is the most reliable way to evaluate transfection efficiency used for normalization purposes.

When choosing or designing a control reporter gene, it is important to keep in mind the following criteria. First, as both reporter genes will be expressed in the same cell, one needs to ensure that the control reporter can be assayed independently of the experimental reporter and that its signal will not be influenced by the conditions used to assay the process of interest (e.g., signaling pathway or cell division). Second, the signal from the control reporter gene needs to be robust and should be significantly different from background signal, especially when measured in a 384-well plate format. It has been our experience that many reporter genes (control or experimental) do not perform as well in 384-well format as would be expected based on the signals observed in cells plated in 96- or 6-well plates.

Control Luciferase Reporters. Luciferase assays (Boeckx, 1984) have become the assay of choice in recent years because of their ease of use, large linear signal range, adaptability to plate reader formats, and technical improvements that have increased their reliability and flexibility. The development of dual-luciferase assays (Grentzmann et al., 1998) has been instrumental in allowing the examination of both experimental and control reporters from the same sample, thereby eliminating the need for separate assays. Dual-luciferase assay systems employ the cotransfection of luciferase constructs from phylogenetically diverse organisms that use different substrates and reaction mechanisms. These differences allow the sequential assay of the two luciferases by taking advantage of the fact that one type of luciferase activity can be individually measured and then specifically 
quenched while the second type of activity is being assayed. The most common commercially available dual-luciferase system uses a beetle (firefly) luciferase and a Renilla (a cnidarian) luciferase (Promega, Madison, WI). Most experimental reporter constructs drive firefly luciferase, whereas Renilla is used in the control construct. Several Renilla-based control reporter genes are commercially available. However, these constructs were designed for use in mammalian systems and the promoters they use do not work well in insect cells. Among them, the thymidine kinase (TK) promoter driven-Renilla, SV40-driven Renilla, and CMV-driven Renilla control vectors give very low signals in 384-well plate luciferase assays when tested in either Drosophila S2 or clone 8 cell lines at $24^{\circ}$. Consequently, we sought to design our own Renilla control constructs for use in Drosophila cell-based HTS assays.

The ideal promoter to drive a Renilla control reporter should be capable of directing high levels of expression without being affected by or affecting the cellular process or signaling pathways that are being targeted in a particular HTS assay. With this criterion in mind, we selected five candidate sequences to drive Renilla expression in a control reporter: a portion of the Drosophila actin5C promoter, the ubiquitin promoter, the mitochondrial large ribosomal protein 49 promoter, the RNA polymerase II 215 subunit promoter (pol II), and the RNA polymerase III 128 subunit promoter (pol III). The first two promoters are known to drive a high level of expression in other heterologous systems, whereas the promoter for mRpL49 (a gene often used as a control in Northern blot analysis of Drosophila cells or tissues) was predicted to lead to a robust expression in most cell types. The two RNA polymerase promoters were selected based on our assumption that, as RNA polymerases are likely to be expressed at high levels in continually dividing cells, their promoters were likely to be highly active in cultured cells.

We tested each of these Renilla constructs for luciferase activity in a 384-well assay, using both Drosophila clone 8 and S2 cells-the cells most often used for transfection assays in our laboratory. As seen in Fig. 2A, the ubiquitin-, mRpL49-, RNA polymerase II-, and SV40-promoter-driven Renilla constructs resulted in signals very similar to the background signal observed with the empty vector alone. Their low levels of expression preclude their use in screens carried out in 384-well plate format. Only the Actin-Renilla and pol III-Renilla vectors gave sufficient signals to be usable in HTS screens. These two Renilla constructs were further tested to ensure that they were not affected by conditions used to study the signaling pathways investigated in our screens. We found that the actin5C-Renilla ectopically transactivated at least one experimental pathway reporter in three signaling pathways tested and was therefore ill suited as a control 
construct in our experiments. The pol III-Renilla had no effect on any of the signaling pathways tested. Testing of the pol III-Renilla also demonstrated that it has a roughly linear dose-response curve in the range used in our 384-well assays (Fig. 2B).

It is important to realize that addition of dsRNA to any transfection assay system reduces the expression of transfected DNA expression constructs between 10 - and 20 -fold, probably by competing with the intake of plasmid DNA by the cells (data not shown). As a result of the general reduction in signals caused by dsRNA and in light of the transactivation effects of actin5C-Renilla that we observed, we settled on the Pol IIIRenilla construct as the optimal control reporter for transfection based assays in 384-well formats. The pol III promoter leads to signals that are consistently 10 -fold above background and are not affected nor interfered with by any of the signaling pathways examined. (Three different pathways have been formally tested.) Although it is imperative to evaluate the behavior of this promoter in the context of every new assay and cell type, we would recommend use of the pol III-Renilla for normalization purposes in transfection assays. This is not to say, however, that other constructs cannot be used for normalization, especially in larger plate formats (Lum et al., 2003). Provided their signals have been adequately studied and remain unchanged under the biological conditions tested in a given assay, other vectors, such as the actin5C-Renilla vector, are also suitable.

Edge Effects. One potential artifact in the process of normalization that we have encountered in the course of luciferase assays is the phenomenon of what has often been called edge effects. The term refers to the observation that artificially high, normalized values are returned for data collected from wells specifically located alongside the edges of a plate. Based on the calculated mean from the other wells in the plate, it appears that the increased value after normalization in these wells is not due to higher firefly luciferase activity. Instead, the edge effect correlates with a reduction in Renilla signal in these wells, and this reduction yields normalized values that are artificially high. We have not been able to determine exactly why edge effects occur or how to reliably prevent them. Edge effects are also observed in visual screens of microscopy images, sometimes manifesting as a decrease in cell density (and possibly morphology) in the outermost wells. The basis for these effects is again unclear, but medium evaporation or uneven treatment of the entire plate surface might be contributing factors. However, edge effects are clearly identifiable in the normalized values and they can be accounted for during the analysis.

Data Analysis. To identify bona fide dsRNA-specific phenotypes, the numerical readouts of the reporter gene assay need to be standardized against the appropriate internal control. To ensure meaningful comparisons 

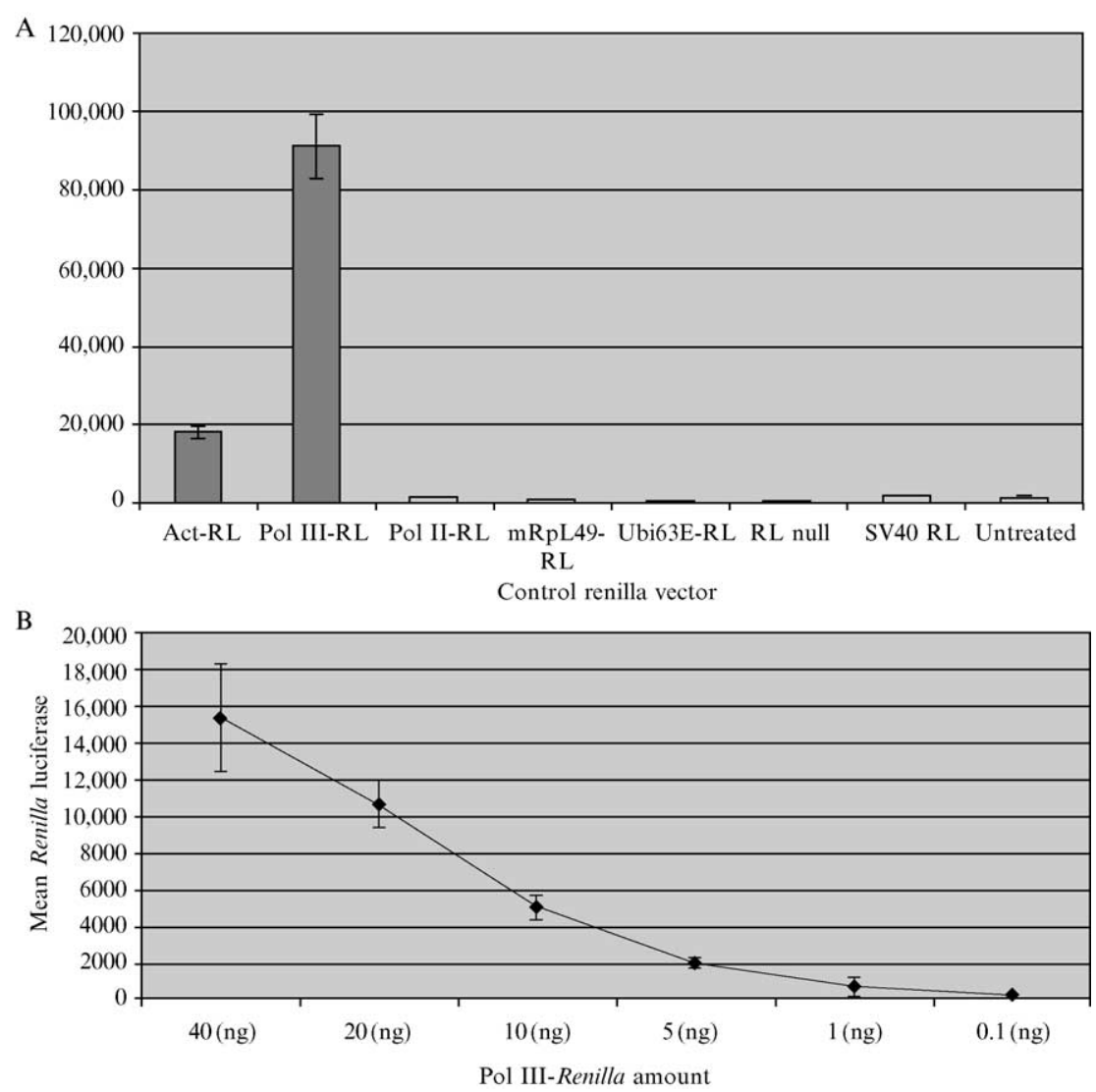

FIG. 2. Analysis of control Renilla reporter constructs. (A) Comparison of luciferase expression. A total of $20 \mathrm{ng}$ of the indicated Renilla luciferase (RL) control reporter construct was transfected along with $90 \mathrm{ng}$ of experimental reporter into 25,000 clone 8 cells in a 384-well plate, using Effectene. RL activity was determined by using the Dual-Glo kit (Promega) and read by a Analyst plate reader (Molecular Devices) after 5 days of transfection. Each treatment represents the average of 8 wells from a 384-well plate. RL-Null is the parental vector (Promega) into which the promotor regions were subcloned, and SV40 RL is a commercially available SV40 promoter-driven Renilla (Promega). All promoter fragments were subcloned into the $B g l \mathrm{II}-\mathrm{Spe} 1$ sites of the $\mathrm{pRL}$-null vector (Promega). Promoter regions corresponding to the pol II and mRpL49 promoters were obtained after PCR amplification of the region between positions 200965 and 200785 for pol II and 282684 and 283154 for mRpl49, according to the genome annotation numbering. The Act5C promoter region is identical to that cloned into the pACW vector available from the DGRC. Details for the pol III construct are available on request. (B) Titration of pol III-Renilla expression. Transfection of decreasing amounts of pol III-Renilla into clone 8 cells demonstrates that Renilla expression is linear from roughly 10 to $40 \mathrm{ng}$ of transfected pol III-Renilla. Transfection was done as in (A) except for the indicated amounts of pol III-Renilla and the presence of $75 \mathrm{ng}$ of green fluorescent protein (GFP) dsRNA in each well. Each treatment represents the average of 6 wells from a 384-well plate. 
between plates, data sets are then mean- or median centered for each individual plate. For each dsRNA condition, the normalized value is assigned a significance or $z$-score value by calculating the number of standard deviations away from the plate median. In this approach, differences between plates are taken into account to calculate the significance of each value, depending on the variability of each assay plate.

Results from duplicate or multiple screens are averaged before threshold selection. The selection of the threshold used to generate a list of candidate targets depends on overall assay parameters, such as signal-to-noise ratio and dynamic range. A good indicator for threshold selection is usually the performance of spiked-in positive controls. In previous genomewide screens, we set a threshold of three standard deviations and above to select with $99.9 \%$ confidence the most statistically significant phenotypes. Data analysis and data representation are usually performed by MATLAB (MathWorks, Nation).

The sequence from each dsRNA that passes the selection threshold is then analyzed for potential off-target effects by BLASTN against all predicted transcripts. Sequences with potential off-site effects are flagged in downstream analysis. The list of predicted gene targets is confirmed by BLASTN searches against the latest published Drosophila genome sequence and mapped to specific chromosomes. The identified genes are searched for associated gene ontology, mutant allele, and RNAi phenotype annotations in FlyBase (http://flybase.bio.indiana.edu/) and other public databases. The predicted protein sequences of genes identified by RNAi phenotypes can then be searched for conserved protein domains by using InterPro.

\section{Perspectives and Conclusions}

In this chapter, we focused on the most basic issues relevant to highthroughput RNAi screens in Drosophila cells in a 384-well plate format. The technology overall is robust and allows the rapid identification of dsRNA candidates that affect a specific readout. In the upcoming years, we expect to see significant improvements and advances in methodology. The range of assays available for this approach will be broadened to include existing readout formats that have not been optimized for the 384-well plate format. Possible applications include cytoblot assays for antibody-based screens, FRET analysis, and HTS multiplexing of mRNA transcripts as signature of a pathway or process. It will also be necessary to develop new cell lines and primary cultures to extend the repertoire of cells in which existing screens can be performed, and, importantly, to enable new screens that address highly specialized functions such as muscle fusion, neuronal outgrowth, or epithelial cell polarity. These advances will require 
the development of increasingly sophisticated software programs that will automatically scan images to identify or quantify user-defined features (e.g., specific cellular structures, protein localization, dynamic processes, or cell size). To better handle the large amount of data returned from these screens and process the growing load of information as more screens get completed, more robust statistical analyses will need to be developed. These analyses will suggest a variety of criteria on which to base our assignment for potential hits, as opposed to the current empirical and static threshold value. Lastly, there will be added flexibility on how to conduct RNAi screens, as alternative platforms (e.g., RNAi cell microarrays) are likely to emerge as powerful methodologies, rivaling existing ones both in speed and economy of reagents.

\section{Acknowledgments}

We thank past and present members of the Perrimon laboratory for discussions on technical issues relevant to RNAi screens in tissue culture cells. K. N. was supported by an NRSA. M. B. was supported by an Emmy-Noether grant from the Deutsche Forschungsgemeinschaft. A. K. was supported by The Jane Coffin Childs Memorial Fund for Medical Research. This work was supported by a grant from the NIGMS and HHMI. N. P. is an investigator of the Howard Hughes Medical Institute.

\section{References}

Adams, M. D., Celniker, S. E. et al. (2000). The genome sequence of Drosophila melanogaster. Science 287(5461), 2185-2195.

Boeckx, R. L. (1984). Chemiluminescence: Applications for the clinical laboratory. Hum. Pathol. 15(2), 104-111.

Boutros, M., Kiger, A. A., Armknecht, S., Kerr, K., Hild, M., Koch, B., Haas, S. A., Consortium, H. F., Paro, R., and Perrimon, N. (2004). Genome-wide RNAi analysis of growth and viability in Drosophila cells. Science 303(5659), 832-835.

Carpenter, A. E., and Sabatini, D. M. (2004). Systematic genome-wide screens of gene function. Nat. Rev. Genet 5(1), 11-22.

Clemens, J. C., Worby, C. A., Simonson-Leff, N., Muda, M., Maehama, T., Hemmings, B. A., and Dixon, J. E. (2000). Use of double-stranded RNA interference in Drosophila cell lines to dissect signal transduction pathways. Proc. Natl. Acad. Sci. USA 97(12), 6499-6503.

Dykxhoorn, D. M., Novina, C. D., and Sharp, P. A. (2003). Killing the messenger: Short RNAs that silence gene expression. Nat. Rev. Mol. Cell Biol. 4(6), 457-467.

Echalier, G., and Ohanessian, A. (1969). Isolation, in tissue culture, of Drosophila melangaster cell lines. C. R. Acad. Sci. Hebd. Seances Acad. Sci. D. 268(13), 1771-1773.

Fire, A. (1999). RNA-triggered gene silencing. Trends Genet. 15(9), 358-363.

Grentzmann, G., Ingram, J. A., Kelly, P. J., Gesteland, R. F., and Atkins, J. F. (1998). A dualluciferase reporter system for studying recording signals. RNA 4(4), 479-486.

Haars, R., Zentgraf, H., Gateff, E., and Bautz, F. A. (1980). Evidence for endogenous reovirus-like particles in a tissue culture cell line from Drosophila melanogaster. Virology 101(1), 124-130. 
Hannon, G. J. (2002). RNA interference. Nature 418(6894), 244-251.

Hild, M., Beckmann, B., Haas, S. A., Koch, B., Solovyev, V., Busold, C., Fellenberg, K., Boutros, M., Vingron, M., Sauer, F., Hoheisel, J. D., and Paro, R. (2003). An integrated gene annotation and transcriptional profiling approach towards the full gene content of the Drosophila genome. Genome Biol. 5(1), R3.

Kelso, R. J., Buszczak, M., Quinones, A. T., Castiblanco, C., Mazzalupo, S., and Cooley, L. (2004). Flytrap, a database documenting a GFP protein-trap insertion screen in Drosophila melanogaster. Nucleic Acids Res. 32, D418-D420. (Database issue).

Kiger, A., Baum, B., Jones, S., Jones, M., Coulson, A., Echeverri, C., and Perrimon, N. (2003). A functional genomic analysis of cell morphology using RNA interference. J. Biol. 2(4), 27.

Lum, L., Yao, S., Mozer, B., Rovescalli, A., Von Kessler, D., Nirenberg, M., and Beachy, P. A. (2003). Identification of hedgehog pathway components by RNAi in Drosophila cultured cells. Science 299(5615), 2039-2045.

McManus, M. T., and Sharp, P. A. (2002). Gene silencing in mammals by small interfering RNAs. Nat. Rev. Genet. 3(10), 737-747.

Paddison, P. J., and Hannon, G. J. (2003). siRNAs and shRNAs: Skeleton keys to the human genome. Curr. Opin. Mol. Ther. 5(3), 217-224.

Peel, D. J., Johnson, S. A., and Milner, M. J. (1990). The ultrastructure of imaginal disc cells in primary cultures and during cell aggregation in continuous cell lines. Tissue Cell 22(5), 749-758.

Schneider, I. (1972). Cell lines derived from late embryonic stages of Drosophila melanogaster. J. Embryol. Exp. Morphol. 27(2), 353-365.

Ui, K., Nishihara, S., Sakuma, M., Togashi, S., Ueda, R., Miyata, Y., and Miyake, T. (1994). Newly established cell lines from Drosophila larval CNS express neural specific characteristics. In Vitro Cell Dev. Biol. Anim. 30A(4), 209-216.

Yanagawa, S., Lee, J. S., and Ishimoto, A. (1998). Identification and characterization of a novel line of Drosophila Schneider S2 cells that respond to wingless signaling. J. Biol. Chem. 273(48), 32353-32359.

\title{
[5] Mechanistic Insights Aid Computational Short Interfering RNA Design
}

\author{
By Queta Boese, Devin Leake, Angela Reynolds, \\ Steven Read, Stephen A. Scaringe, \\ William S. Marshall, and Anastasia Khvorova
}

\begin{abstract}
RNA interference is widely recognized for its utility as a functional genomics tool. In the absence of reliable target site selection tools, however, the impact of RNA interference (RNAi) may be diminished. The primary determinants of silencing are influenced by highly coordinated RNA-protein interactions that occur throughout the RNAi process, including short interfering RNA (siRNA) binding and unwinding followed
\end{abstract}

